\title{
Clima de seguridad del paciente en cuatro servicios quirúrgicos de Santander
}

\section{Patient Safety Climate in four Santander surgical units}

\author{
Dairon-Alexander Fonseca-Mesa ${ }^{1 \mathbb{D}} ;$ Piedad-Cecilia Serpa-Pérez ${ }^{1}{ }^{\mathbb{D}} ;$ José-Hugo Arias-Botero $^{2}$ (D)
}

Forma de citar: Fonseca-Mesa DA, Serpa-Pérez PC, Arias-Botero JH. Clima de seguridad del paciente en cuatro servicios quirúrgicos de Santander. Salud UIS. 53: e21006. doi: https://doi.org/10.18273/saluduis.53.e:21006 (c) (1)

\section{Resumen}

Introducción: conocer la percepción del clima de seguridad al interior de los servicios quirúrgicos es una tarea importante para establecer acciones que reduzcan el riesgo de eventos adversos durante la atención del paciente. Objetivo: Caracterizar el clima de seguridad en los servicios quirúrgicos de cuatro instituciones de salud de Santander. Materiales y métodos: Estudio observacional de corte transversal. Se aplicó el instrumento Hospital Survey on Patient Safety. Los resultados se reportaron como los promedios de repuestas positivas y negativas. Se analizaron las características asociadas a las dimensiones con mayores puntajes de percepción negativa. Resultados: participaron 164 trabajadores. Las dimensiones con mayor percepción positiva fueron prácticas seguras $(84 \%)$, dotación de personal $(83,2 \%)$ y aprendizaje organizacional $(78,4 \%)$. Las dimensiones con percepción desfavorable fueron respuesta no punitiva al error $(40,2 \%)$, carga de trabajo $(51,7 \%)$, comunicación $(54,3 \%)$ y transiciones y transferencias entre servicios $(58,1 \%)$. Se encontró asociación entre una baja percepción en las transiciones y transferencias entre servicios con la contratación por prestación de servicios y con el cargo Médico Especialista y Anestesiólogo. Los bajos puntajes en comunicación se asociaron a la contratación gremial. No se encontraron factores asociados para las dimensiones respuesta no punitiva al error y carga de trabajo. Conclusiones: La percepción sobre clima seguridad en general es considerada positiva, aunque existen oportunidades de mejora. Los factores como tipo de contratación y cargo se asociaron a una percepción negativa de clima de seguridad. Los trabajadores se sienten juzgados al reportar eventos adversos.

Palabras clave: Gestión de la Calidad; Seguridad del Paciente; Clima de Seguridad; Eventos Adversos; Unidades Quirúrgicas; Cultura de Seguridad; Percepción.

\begin{abstract}
Introduction: Knowing the perception of the safety climate within surgical services has become an important task to establish actions that reduce the risk of suffering adverse events during patient care. Objective: To characterize the safety climate in the surgical services of four health institutions in Santander. Materials and methods: Observational

1. Universidad de Santander. Bucaramanga, Colombia.

2. Universidad CES. Medellín, Colombia.

Correspondencia: Dairon Alexander Fonseca Mesa. Dirección: UDES Cra. 38 41-74 Bucaramanga. Teléfono: +7 3144498358. Correo electrónico: buc18801001@mail.udes.edu.co
\end{abstract}


cross-sectional study. The Hospital Survey on Patient Safety instrument was applied. The results were reported as the means of positive and negative responses. The characteristics associated with the dimensions with the highest negative perception scores were analyzed. Results: 164 workers participated. The dimensions with the highest positive perception were safe practices $(84 \%)$, staffing $(83.2 \%)$ and organizational learning $(78.4 \%)$; Dimensions with unfavorable perception were non-punitive response to error $(40.2 \%)$, workload $(51.7 \%)$, communication $(54.3 \%)$ and transitions and transfers between services (58.1\%). Association between a low perception in transitions and transfers between services with contracting for the provision of services and with the position of Specialist (Physician and Anesthesiologist) was found. Low scores in communication were associated with union hiring. No associated factors were found for the non-punitive response to error and workload dimensions. Conclusions: In general, the perception of safety climate is considered positive, although there are opportunities for improvement. Factors such as type of hiring and position were more associated with negative perception. Workers feel judged when reporting adverse events.

Keywords: Quality management; Patient safety; Safety climate; Adverse Events; Operating Rooms; Safety Culture; Perception.

\section{Introducción}

Los eventos adversos pueden originarse en diferentes procesos de la institución como, por ejemplo, el área administrativa, en donde se gestionan y coordinan los recursos y el talento humano. En el área asistencial se encuentran los relacionados con el trabajo en equipo y la comunicación ${ }^{1}$, el registro de historias clínicas, la administración de medicamentos o hemocomponentes, la recepción y análisis de muestras de laboratorio o patología, procedimientos quirúrgicos y la anestesia ${ }^{2}$, las infecciones nosocomiales, el transporte intrahospitalario de pacientes ${ }^{3,4}$, los errores ocasionados por equipos o insumos biomédicos, los relacionados con la infraestructura de la institución, o los que son proporcionales al estado clínico del paciente, sus comportamientos o creencias $^{5}$. Estudios recientes demuestran que las diferentes áreas, tanto asistenciales o administrativas, pueden afectar de manera directa $o$ indirecta al paciente, y la probabilidad de presentarse un evento adverso es elevada si no se cumple con las normas y protocolos estandarizados por la institución.

En los servicios quirúrgicos se presenta una gran cantidad de eventos adversos, la mitad de ellos son considerados prevenibles ${ }^{6}$. Dentro de sus principios, la mayoría de las instituciones quirúrgicas buscan dirigir, mejorar y evaluar el desempeño de sus intervenciones, enfocados en un modelo de calidad, atención integral y aptitudes desarrolladas en el marco del ejercicio ético profesional, para lograr la satisfacción de sus usuarios y una evolución quirúrgica favorable ${ }^{7,8}$. De tal modo podrán proporcionar a la población una atención centrada en sus principales necesidades de salud, basada en criterios de mejora continua y fomentar un trato humanístico en un entorno de confianza y seguridad ${ }^{9,10}$.
El servicio de quirófanos proporciona una atención altamente compleja, en este entorno, existen mayores posibilidades de que se generen acciones inseguras que contribuyen a la ocurrencia de eventos adversos graves que aumentan los costos de no calidad, la probabilidad de muerte o secuelas permanentes y la afectación de la credibilidad de las instituciones ${ }^{2}$. Estas situaciones aumentan cuando no se cuenta con un sistema de calidad fortalecido. En este sentido, las organizaciones de salud deben comprometerse a implementar procesos y acciones encaminadas a reducir la incidencia de eventos adversos e integrar a sus colaboradores al plan de mejoramiento continuo institucional con acciones que consolidan la cultura de la seguridad ${ }^{11-13}$. Existen pocos estudios enfocados en medir la percepción del clima de seguridad del paciente en las unidades quirúrgicas ${ }^{14}$.

Se considera que el clima de seguridad permite, a través de la medición de actitudes y percepciones individuales de los colaboradores, evaluar la cultura de seguridad del paciente. La medición periódica de la percepción de clima de seguridad, permite monitorizar la trazabilidad de los indicadores de calidad implementados en cada institución ${ }^{15}$. Así mismo, un aumento en las percepciones positivas sobre el clima de seguridad, permite evidenciar la eficacia de las acciones implementadas para consolidar una cultura de seguridad del paciente.

La escasa disponibilidad de evidencia en la región sobre la percepción del clima de seguridad del paciente en el personal de las unidades quirúrgicas y la necesidad de implementar estrategias que fortalezcan la gestión y las buenas prácticas en estos servicios, justificó la realización de este estudio, que tuvo como objetivo 
caracterizar la percepción del clima de seguridad del paciente en los servicios quirúrgicos aplicando el instrumento "Hospital Survey on Patient Safety"16, validado en Colombia para unidades quirúrgicas ${ }^{17}$.

\section{Material y métodos}

Estudio observacional de corte transversal con interés analítico. Se aplicó la encuesta Hospital Survey on Patient Safety desarrollado por la Agency for Healthcare Research and Quality (AHRQ) ${ }^{16}$, validada para servicios quirúrgicos en Colombia ${ }^{17}$. Esta encuesta se aplica de manera auto administrada y consta de 60 ítems relativos al clima de seguridad los cuales están agrupados en 10 dimensiones. Las preguntas tienen cinco opciones de respuesta, organizadas en una escala tipo Likert: de 1 (muy en desacuerdo o nunca) a 5 (muy de acuerdo o siempre); estas respuestas se organizan en tres categorías: negativo, neutral o positivo, de acuerdo con la guía del desarrollador del instrumento ${ }^{18}$.

Se incluyó a todo el personal asistencial y administrativo de cuatro instituciones de salud de mediana y alta complejidad de atención de la ciudad de Bucaramanga y su área Metropolitana que se desarrollaban actividades relacionadas con el funcionamiento operativo de la unidad quirúrgica y con tiempo de permanencia en el servicio mayor a tres meses. Se excluyó al personal que trabajaba específicamente en el área de calidad y aquellos que no desarrollaban actividades relacionadas con el funcionamiento operativo del área quirúrgica de la institución (mantenimiento), y los que no deseaban participar en la investigación. Se incluyeron instituciones con volúmenes de cirugía mayores a 200 procedimientos por mes. No se incluyeron instituciones que atendían una sola especialidad quirúrgica (ej. obstétrica, oftalmológica).

Se solicitó autorización a los desarrolladores del instrumento para su uso. El estudio fue aprobado por los Comités de Ética de Investigación de las cuatro instituciones de salud participantes. Se obtuvo consentimiento por escrito de los participantes.
Para la descripción de las características de las unidades quirúrgicas se presentan frecuencias absolutas $\mathrm{y}$ relativas, las variables cualitativas y mediana con rango intercuartílico y las variables cuantitativas.

Para el análisis de las variables del instrumento que mide cultura de la seguridad se realizó una descripción de las respuestas a los ítems en frecuencias absolutas y relativas, las categorías se agruparon en: "Negativo", "Neutral" y "Positivo" como se describió anteriormente. Para cada dimensión, se calculó el porcentaje promedio de respuestas positivas de los ítems que componen la dimensión, los estimadores fueron calculados con su intervalo de confianza del $95 \%$.

Para analizar las dimensiones con oportunidades de mejora, se calculó el porcentaje promedio de respuestas negativas. Se compararon las características sociodemográficas y laborales entre quienes tenías más de $50 \%$ y menos de $50 \%$ de respuestas negativas, se utilizó prueba de Chi2 para variables cualitativas y U de Mann Whitney para variables cuantitativas. Se consideró significativo un valor de $\mathrm{p}$ menor o igual a 0,05 .

Se analizó la asociación de las características del personal con la percepción negativa (trabajadores de las unidades con más de $50 \%$ de respuesta negativas) utilizando la Razón de Prevalencias la cual se reporta con su intervalo de confianza (IC) $95 \%$. Para los análisis se utilizó el software Stata $^{\circledR}$ versión 12 (StataCorp).

\section{Resultados}

Tres instituciones eran de carácter privado y una pública. Dos instituciones participantes estaban acreditadas y tres prestan servicios de salud de III nivel de atención. Con una tasa de respuesta del 56,5\% a la encuesta. Las características de las instituciones participantes se presentan en la Tabla 1.

Tabla 1. Características de las instituciones.

\begin{tabular}{ccccccc}
\hline Institución & Característica & $\begin{array}{c}\text { Tipo } \\
\text { complejidad }\end{array}$ & $\begin{array}{c}\text { Total de } \\
\text { camas }\end{array}$ & $\begin{array}{c}\text { Total de } \\
\text { Quirófanos }\end{array}$ & $\begin{array}{c}\text { Encuestas } \\
\text { aplicadas }\end{array}$ & $\begin{array}{c}\text { Tasa de } \\
\text { respuesta }\end{array}$ \\
\hline A & Privada & Alta & 211 & 5 & 69 & $82 \%$ \\
B & Privada & Alta & 214 & 8 & 49 & $40,8 \%$ \\
C & Privada & Alta & 198 & 6 & 27 & $45 \%$ \\
D & Pública & Media & 70 & 2 & 19 & $73 \%$ \\
\hline
\end{tabular}

Fuente: Elaboración de los autores. 
Se obtuvo respuesta de 164 participantes. Se contó con mayor participación de auxiliares de enfermería, instrumentación quirúrgica, enfermería y anestesiología; en su mayoría, población femenina. Se identificó que la mayor parte de los encuestados están contratados como planta de cargo o término indefinido. Algunas de las instituciones estudiadas tienen mayor capacidad de respuesta ante la demanda quirúrgica de pacientes, además, de la capacidad de talento humano contratado para prestar adecuadamente el servicio (Tabla 2).
La institución $\mathrm{C}$, cuenta con mayor permanencia de los colaboradores en la institución y en el servicio de cirugía. La mayoría de los participantes tenían relación directa con el paciente. El 50\% de los colaboradores que están contratados a término indefinido laboran 48 horas semanales o menos; el trabajo lo realizan en turnos rotativos de 8 a 12 horas. (Tabla 2).

Tabla 2. Características de los participantes.

\begin{tabular}{|c|c|c|c|c|}
\hline Institución & $\mathbf{A}$ & B & $\mathbf{C}$ & D \\
\hline Tiempo de trabajo en la institución/unidad & $\mathrm{Me}(\mathrm{RIQ}) *$ & $\mathrm{Me}(\mathrm{RIQ})^{*}$ & $\mathrm{Me}(\mathrm{RIQ}) *$ & $\mathrm{Me}(\mathrm{RIQ})^{*}$ \\
\hline Tiempo en la institución en años & $4(1,25-6)$ & $2(0,7-3)$ & $3(8-12)$ & $5(2-18)$ \\
\hline Tiempo en la unidad en años & $4(1-6)$ & $1(0,37-3)$ & $3(8-10)$ & $3(2-5)$ \\
\hline Horas a la semana & $48(48-48)$ & $48(48-48)$ & $48(48-48)$ & $48(48-48)$ \\
\hline Años de trabajo en la profesión. & $10(5-15)$ & $3(2-10)$ & $8(4-16)$ & $6(3-20)$ \\
\hline Cargo & n $(\%)$ & n $(\%)$ & n $(\%)$ & n $(\%)$ \\
\hline Auxiliar de Enfermería & $27(39,1)$ & $17(34,7)$ & $9(33,3)$ & $7(36,8)$ \\
\hline Instrumentador Quirúrgico & $9(13)$ & $9(18,4)$ & $4(14,8)$ & $3(15,8)$ \\
\hline Médico general & $2(2,9)$ & $4(8,2)$ & & $2(10,5)$ \\
\hline Anestesiólogo & $5(7,2)$ & $2(4,1)$ & $3(11,1)$ & $1(5,3)$ \\
\hline Enfermera profesional & $5(7,2)$ & $7(14,3)$ & $5(18,5)$ & $2(10,5)$ \\
\hline Médico Especialista & $4(5,8)$ & & & $1(5,3)$ \\
\hline Servicios generales & $4(5,8)$ & $2(4,1)$ & $2(7,4)$ & $2(10,5)$ \\
\hline Farmacéutico & $3(4,3)$ & $4(8,2)$ & $4(14,8)$ & \\
\hline Auxiliar administrativo & $6(8,7)$ & $2(4,1)$ & & \\
\hline Administración/dirección & $3(4,3)$ & $1(2,0)$ & & \\
\hline Ingeniero Biomédico & $1(1,4)$ & & & \\
\hline Camillero & & $1(2,0)$ & & $1(5,3)$ \\
\hline Área de trabajo & n $(\%)$ & n (\%) & n $(\%)$ & n $(\%)$ \\
\hline Cirugía & $48(69,6)$ & $39(79,6)$ & $19(70,4)$ & $12(63,2)$ \\
\hline Anestesiología & $5(7,2)$ & $2(4,1)$ & $3(11,1)$ & $1(5,3)$ \\
\hline Obstetricia & $1(1,4)$ & & & $5(26,3)$ \\
\hline Esterilización & $2(2,9)$ & $1(2,0)$ & $1(3,7)$ & $1(5,3)$ \\
\hline Farmacia & $3(4,3)$ & $4(8,2)$ & $4(14,8)$ & \\
\hline Administrativo & $9(13,0)$ & $3(6,1)$ & & \\
\hline Equipos biomédicos & $1(1,4)$ & & & \\
\hline Contacto con los pacientes & n $(\%)$ & n (\%) & n (\%) & n (\%) \\
\hline $\mathrm{Si}$ & $52(75,4)$ & $43(87,8)$ & $20(74,1)$ & $16(84,2)$ \\
\hline No & $17(24,6)$ & $6(12,2)$ & $7(24,9)$ & $3(15,8)$ \\
\hline Tipo de contrato & n $(\%)$ & n $(\%)$ & n $(\%)$ & n $(\%)$ \\
\hline Prestación de servicios & $11(15,9)$ & & $2(7,4)$ & $1(5,3)$ \\
\hline Planta de cargos & $57(82,6)$ & $49(100)$ & $25(92,6)$ & $3(15,8)$ \\
\hline Asociación gremial & & & & $15(78,9)$ \\
\hline Aprendizaje & $1(1,4)$ & & & \\
\hline
\end{tabular}

Fuente: Elaboración de los autores.

Puntaje de respuestas positivas por dimensión: Entre las instituciones se observaron diferencias en los puntajes para todas las dimensiones evaluadas con porcentaje de respuesta positiva $(\geq 75 \%)$. Las dimensiones con la mayor percepción positiva fueron, prácticas seguras $84 \%$ (IC95\%: 78,4-89,6), insumos, equipos y dispositivos 83,2\% (IC95\%: 77,5-88,9) y aprendizaje organizacional 78,4\% (IC 95\%: 72,1-84,7). 
La institución A, fue quien más tuvo fortalezas dentro las dimensiones evaluadas, seguido de la institución $\mathrm{C} \mathrm{y}$ $\mathrm{B}$, respectivamente. Por otra parte, se evidencia que las instituciones A, B y C comparten la fortaleza de contar con la suficiencia de insumos, equipos y dispositivos médicos para realizar intervenciones seguras (Tabla 3).

Tabla 3. Respuestas positivas por dimensión.

\begin{tabular}{|c|c|c|c|c|c|}
\hline Institución & $\mathbf{A}$ & B & $\mathbf{C}$ & D & TOTAL \\
\hline Dimensión & $n=69$ IC95\% & $n=49$ IC95\% & $n=27$ IC95\% & $n=19$ IC95\% & $n=164 \mathrm{IC} 95 \%$ \\
\hline Trabajo en equipo dentro del área & $\begin{array}{c}80,29 \% \\
(70,90-89,68)\end{array}$ & $\begin{array}{c}60,41 \% \\
(46,72-74,10)\end{array}$ & $\begin{array}{c}71,85 \% \\
(54,89-88,82)\end{array}$ & $\begin{array}{c}69,47 \% \\
(48,77-90,18)\end{array}$ & $\begin{array}{c}71,7 \% \\
(64,8-78,6)\end{array}$ \\
\hline Carga de trabajo & $\begin{array}{c}58,33 \% \\
(46,70-69,97)\end{array}$ & $\begin{array}{c}45,92 \% \\
(31,97-59,87)\end{array}$ & $\begin{array}{c}56,48 \% \\
(37,78-75,18)\end{array}$ & $\begin{array}{c}35,53 \% \\
(14,01-57,05)\end{array}$ & $\begin{array}{c}51,7 \% \\
(44,1-59,3)\end{array}$ \\
\hline $\begin{array}{l}\text { Aprendizaje organizacional y } \\
\text { mejoramiento continuo }\end{array}$ & $\begin{array}{c}86,96 \% \\
(79,01-94,90)\end{array}$ & $\begin{array}{c}71,72 \% \\
(59,11-84,33)\end{array}$ & $\begin{array}{c}87,30 \% \\
(74,74-99,86)\end{array}$ & $\begin{array}{c}57,89 \% \\
(35,69-80,10)\end{array}$ & $\begin{array}{c}78,4 \% \\
(72,1-84,7)\end{array}$ \\
\hline Respuesta no punitiva al error & $\begin{array}{c}44,93 \% \\
(33,19-56,66)\end{array}$ & $\begin{array}{c}34,18 \% \\
(20,90-47,46)\end{array}$ & $\begin{array}{c}36,11 \% \\
(17,99-54,23)\end{array}$ & $\begin{array}{c}44,74 \% \\
(22,38-67,09)\end{array}$ & $\begin{array}{c}40,2 \% \\
(32,7-44,7)\end{array}$ \\
\hline $\begin{array}{l}\text { Expectativas y acciones del } \\
\text { supervisor o del administrador que } \\
\text { promueven la seguridad }\end{array}$ & $\begin{array}{c}82,61 \% \\
(73,67-91,55)\end{array}$ & $\begin{array}{c}63,27 \% \\
(49,77-76,76)\end{array}$ & $\begin{array}{c}75,31 \% \\
(59,04-91,57)\end{array}$ & $\begin{array}{c}54,39 \% \\
(31,99-76,78)\end{array}$ & $\begin{array}{c}72,4 \% \\
(65,6-79,2)\end{array}$ \\
\hline $\begin{array}{l}\text { Comunicación abierta y } \\
\text { retroalimentación }\end{array}$ & $\begin{array}{c}54,49 \% \\
(42,74-66,24)\end{array}$ & $\begin{array}{c}58,37 \% \\
(44,57-72,17)\end{array}$ & $\begin{array}{c}60,0 \% \\
(41,52-78,48)\end{array}$ & $\begin{array}{c}34,74 \% \\
(13,33-56,15)\end{array}$ & $\begin{array}{c}54,3 \% \\
(44,7-61,9)\end{array}$ \\
\hline Frecuencia de reporte de eventos & $\begin{array}{c}59,42 \% \\
(47,83-71,01)\end{array}$ & $\begin{array}{c}65,99 \% \\
(52,72-79,25)\end{array}$ & $\begin{array}{c}72,84 \% \\
(56,06-89,62)\end{array}$ & $\begin{array}{c}42,11 \% \\
(19,90-64,31)\end{array}$ & $\begin{array}{c}61,6 \% \\
(54,2-69)\end{array}$ \\
\hline Transiciones & $\begin{array}{c}63,04 \% \\
(51,65-74,43)\end{array}$ & $\begin{array}{c}52,30 \% \\
(38,31-66,28)\end{array}$ & $\begin{array}{c}66,67 \% \\
(48,89-84,45)\end{array}$ & $\begin{array}{c}42,76 \% \\
(20,52-65,01)\end{array}$ & $\begin{array}{c}58,1 \% \\
(50,5-65,7)\end{array}$ \\
\hline $\begin{array}{l}\text { Insumos equipos y dispositivos } \\
\text { médicos }\end{array}$ & $\begin{array}{c}92,93 \% \\
(86,89-98,98)\end{array}$ & $\begin{array}{c}77,30 \% \\
(65,57-89,03)\end{array}$ & $\begin{array}{c}90,74 \% \\
(79,81-100)\end{array}$ & $\begin{array}{c}51,97 \% \\
(29,51-74,44)\end{array}$ & $\begin{array}{c}83,2 \% \\
(77,5-88,9)\end{array}$ \\
\hline Prácticas seguras & $\begin{array}{c}87,86 \% \\
(80,16-95,57) \\
\end{array}$ & $\begin{array}{c}86,73 \% \\
(77,24-96,23) \\
\end{array}$ & $\begin{array}{c}91,67 \% \\
(81,24-100) \\
\end{array}$ & $\begin{array}{c}51,97 \% \\
(29,51-74,44) \\
\end{array}$ & $\begin{array}{c}84 \% \\
(78,4-89,6) \\
\end{array}$ \\
\hline
\end{tabular}

Fuente: Elaboración de los autores.

Porcentaje de respuestas negativas: Entre las dimensiones con mayor número de percepciones negativas ( $\geq 50 \%$ ), están: Carga de trabajo 51,7\% (IC95\%: $44,1-59,3)$, Comunicación abierta y retroalimentación $54,3 \%$ (IC95\%: 44,7-61,9), Transiciones y transferencias entre servicios 58,1\% (IC95\%: 50,5-65,7), y Respuesta no punitiva al error 40,2\% (IC95\%: 32,7-44,7), esta última con mayor representación. La institución D tiene el mayor número de percepciones negativas según los resultados obtenidos en cada dimensión (Tabla 4).

Tabla 4. Percepción de dimensiones con respuestas negativas (oportunidad de mejora).

\begin{tabular}{lc}
\hline \multicolumn{1}{c}{ Dimensión } & Me (RIQ) \\
\hline Trabajo en equipo & $0(0-20)$ \\
Expectativas y acciones del supervisor & $0(0-25)$ \\
Aprendizaje organizacional-mejoramiento & $0(0-14,3)$ \\
continuo & $20(0-20)$ \\
Comunicación abierta y retroalimentación & $0(0-0)$ \\
Frecuencia de reporte de incidentes & $25(0-50)$ \\
Carga de trabajo & $12,5(0-25)$ \\
Transiciones y transferencias entre servicios & $25(0-50)$ \\
Respuesta no punitiva al error & $0(0-0)$ \\
Insumos, equipos y dispositivos médicos & $0(0-0)$ \\
Prácticas seguras &
\end{tabular}

Fuente: Elaboración de los autores.
Análisis de asociación: La categoría de asociación gremial tiene mayor percepción negativa en las dimensiones aprendizaje organizacional y mejoramiento continuo (RP: 5,96, IC95\%: 1,03-22,4), expectativas y acciones del supervisor o del administrador que promueven la seguridad (RP: 2,9, IC95\%: 1,02-6,35), comunicación abierta y retroalimentación (RP: 5,96, IC95\%: 1,83-13,35), frecuencia de reporte de eventos (RP: 3.19 IC95\%: 1.24-5.99), transiciones y transferencias entre servicios (RP: 2,44 IC95\%: 0,7-6,16), e insumos, equipos y dispositivos médicos (RP: 2,86, IC95\%: 1,41-3,13); así mismo, en transiciones y transferencias entre servicios se encontró, además, una asociación en la percepción negativa en los colaboradores contratados por prestación de servicios (RP: 3,25 IC95\%: 1.1-6,96). No obstante, cabe resaltar que el $100 \%$ de las personas con tipo de contrato asociación gremial hacen parte de la institución $\mathrm{D}$, que a su vez fue la institución con menor porcentaje de respuestas positivas (Tabla 5). 
Tabla 5. Razón de Prevalencia.

$>\mathbf{5 0} \%$

Expectativas
Tipo de contrato
Planta de cargos
Prestación de servicios
Asociación gremial
Aprendizaje organizacional
Tipo de contrato
Planta de cargos
Prestación de servicios
Asociación gremial

Comunicación abierta y retroalimentacion

Tipo de contrato

Planta de cargos

Prestación de servicios

Asociación gremial

Frecuencia de reporte de incidentes

Sexo

Femenino

Masculino

Tipo de contrato

Planta de cargos

Prestación de servicios

Asociación gremial

Transiciones y transferencias entre servicios

Cargo

Auxiliar de enfermería

Instrumentador Qx.

Médico general

Anestesiólogo

Enfermera profesional

Médico Especialista

Servicios generales

Farmacéutico

Asist. Administrativo

Otros

Tipo de contrato

Planta de cargos

Prestación de servicios

Asociación gremial

Respuesta no punitiva al error

Cargo

Auxiliar de enfermería

Instrumentador Qx.

Médico general

Anestesiólogo

Enfermera profesional

Médico Especialista

Servicios generales

Farmacéutico

Asist. Administrativo

Otros

Insumos equipos y dispositivos médicos

Tipo de contrato

Planta de cargos

Prestación de servicios

Asociación gremial

Practicas seguras

Tipo de contrato

Planta de cargos

Prestación de servicios

Asociación gremial

Fuente: Elaboración de los autores

$\begin{array}{ccc}12(75) & 122(82,4) & 1 \\ 0 & 15(10,1) & \\ 4(25) & 11(7,4) & 2,\end{array}$

1

2,9

$[1,02-6,35]$

$3(60)$
0

$131(82,4)$

$15(9,4)$

$13(8,2)$

5,96

$[1,03-22,4]$

2 (40)

$6(54,5)$

$1(9,1)$

$4(36,4)$

$128(83,7)$

$14(9,2)$

$11(7,2)$

5,96

[1,83-13,35]

$10(50)$

$107(74,3)$

$37(25,7)$

1

$10(50)$

$120(83,3)$

$14(70)$

$14(9,7)$

$10(6,9)$

$1 \%)$
$5(25)$

$4(22,2)$

0

$56(38,4)$

$25(17,1)$

$1(5,6)$

$4(22,2)$

$4(22,2)$

$1(5,6)$

0

$1(5,6)$

0

$11(61,1)$

$4(22,2)$

$3(16,7)$

$123(84,2)$

$1(1,9)$

$1(33,3)$

$2(66,7)$
$7(4,8)$

$784,8)$

$15(10,3)$

$2(1,4)$

$9(6,2)$

$11(7,5)$

$7(4,8)$

$7(4,8)$

$11(7,5)$

$12(8,2)$

$41(36,6)$

19 (17)

$6(5,4)$

$9(8)$

9 (8)

0

$8(7,1)$

$6(5,4)$

$8(7,1)$

$6(5,4)$

$133(82,6)$

$15(9,3)$

$13(8,1)$

2,86

[1,41-3,13]

[0,21-8,91]

[1,56-11,06]

[0,84-8,16]

[2,42-13,82]

[0,17-7,89]

[0,21-8,91]

[1,1-6,96]

[0,7-6,16]

2,44

1

0,76

0,79

0,57

1,66

0,63

1,44

0,45

[0,3-1,51]

[0,18-2,03]

[0,13-1,68]

[0,88-2,4]

[0,14-1,78]

[0,58-2,38]

[0,06-1,89]

$134(84,3)$

$15(9,4)$

$10(6,3)$ 
En la dimensión Transiciones y transferencias entre servicios, se encontró asociación de respuestas negativas con el cargo, en las categorías de Médico especialista (RP: 9, IC95\%: 2,42-13,82) y Anestesiología (RP: 5,45 , IC95\%: 1,56-11,06), cuando se compara con el personal Auxiliar de enfermería. Para algunas categorías de cargo, no se pudo calcular asociación por la baja cantidad de muestra en la categoría, lo que generó ceros en las casillas de las tablas cruzadas (Tabla 5).

\section{Discusión}

Las mediciones de clima de seguridad del paciente son importantes teniendo en cuenta que permiten una aproximación cuantitativa a la cultura de la seguridad. Esta información se convierte en un insumo importante para el desarrollo de las políticas de seguridad del paciente encaminadas a disminuir la frecuencia de eventos adversos ${ }^{19}$. Mediante la aplicación de la encuesta AHRQ validada para Colombia, este estudio logró identificar la percepción sobre el clima de seguridad en el área de cirugía de cuatro instituciones de Bucaramanga y su área Metropolitana.

Se identificó que la percepción sobre el clima de seguridad varía entre las instituciones participantes, lo que sugiere fases diferentes de consolidación de la cultura de la seguridad, esto implica que las mediciones siempre deberían analizarse para cada institución e incluso, para cada servicio y así evitar las mediciones agrupadas, que no permitirían identificar tales diferencias.

En general las dimensiones Aprendizaje organizacional y mejoramiento continuo, Insumos equipos y dispositivos médicos y Prácticas seguras fueron calificadas como fortalezas con porcentajes de respuesta positiva $78,4 \%, 83,2 \%$ y $84 \%$, respectivamente. La dimensión carga de trabajo, fue valorada como debilidad, dando referencia a la existencia de sobrecarga laboral, lo que puede provocar un incremento en la probabilidad de presentarse un evento adverso. Las dimensiones restantes fueron catalogadas, de manera general, como debilidad. Los puntajes más bajos los tienen respuesta no punitiva al error con un $40,2 \%$, carga de trabajo con un $51,7 \%$, comunicación abierta y retroalimentación con un $54,3 \%$ y transiciones con un $58,1 \%$. Se evidenció además que los trabajadores asistenciales tuvieron una percepción más negativa. Estos resultados coinciden con los reportados en Brasil $^{20}$, que evaluaron el clima de seguridad en tres instituciones de alta complejidad, encontrando que la dimensión de Respuestas no punitivas al error tenía un porcentaje de repuesta positiva del $21,9 \%$ catalogándola como una debilidad; Por el contrario, las dimensiones trabajo en equipo dentro de las unidades hospitalarias, expectativas y acciones para promover la seguridad y aprendizaje organizacional fueron catalogadas como fortalezas.

Así mismo, en Teresina, estado do Piauí (Brasil) ${ }^{21}$, se realizó un estudio de tipo corte transversal en miembros del equipo de enfermería de un centro quirúrgico, para analizar la cultura de la seguridad del paciente. En dicho estudio, las dimensiones con los porcentajes más altos de respuesta positiva fue aprendizaje organizacional y mejora continua $(58,7 \%)$ y la percepción general de seguridad del paciente $(47,1 \%)$. La dimensión de comunicación abierta y retroalimentación, y respuesta punitiva al error, obtuvieron la percepción más negativa $(32,3 \%)$ y $(37 \%)$.

Una revisión sistemática realizada en Irán ${ }^{22}$ evaluó trabajos científicos realizados en diferentes hospitales iraníes donde se aplicó la encuesta "Hospital Survey on Patient Safety" (HSOPSC); en total los investigadores encontraron 27 estudios. Se encontró que las calificaciones de las respuestas para las diferentes dimensiones de la encuesta oscilaron entre $37,79 \%$ y $65,43 \%$. En términos generales, la dimensión con calificación más baja fue la de respuesta no punitiva al error con una media de $37,79 \%$; mientras que la dimensión con puntaje más alto fue aprendizaje organizacional y mejora continua $(65,43 \%)$. Como conclusión, los resultados mostraron un clima de seguridad del paciente bajo que requiere una intervención rápida por parte de los diferentes directivos de las instituciones.

En Almería (España) ${ }^{23}$, un estudio sobre "Percepción de la cultura de seguridad del paciente en enfermeros de atención primaria" aplicó la encuesta de la AHRQ a 201 enfermeros. Para la interpretación de los resultados tomaron las puntuaciones mayores a tres como percepción positiva y mayor a cuatro como percepción claramente positiva. La calificación global sobre la calidad tuvo una puntuación de 3,4 y seguridad del paciente de manera global de 2,97. La dimensión con mejor puntuación fue la categoría de aspectos relacionados con la seguridad del paciente y calidad $(4,13)$, seguida de intercambio de la información con otros servicios asistenciales $(4,18)$ y trabajo en equipo $(4,01)$. La encueta aplicada no consideraba la dimensión respuesta punitiva ante el error, por lo cual no se pudo realizar un comparativo en esta categoría. 
Un estudio realizado en el Hospital Universitario en Alicante ${ }^{24}$, evidenció que ninguna de las dimensiones fue catalogada, en general, como fortaleza. Lo que contrasta con los resultados obtenidos en este estudio, en el que se identificaron percepciones positivas en algunas dimensiones. Las dimensiones que obtuvieron un mayor porcentaje de respuesta positiva son las expectativas y acciones de la dirección/supervisión que favorecen la seguridad (67\%), y trabajo en equipo dentro de la unidad/servicio $(64,6 \%)$. Los resultados obtenidos en ese estudio reportaron una tasa de respuesta del $35,36 \%$, muy baja en relación con la media esperada $(50 \%)$ y a la obtenida en este proyecto de investigación, lo cual podría estar relacionado con diferencias en las características de la y cultura de seguridad implementadas por la institución. En otro estudio realizado en Zaragoza (España) ${ }^{25}$, con tasa de respuesta de $84,6 \%$, la dimensión con mayor percepción positiva fue trabajo en equipo $(64,7 \%)$; las demás dimensiones fueron catalogadas como percepción desfavorable, entre estas, percepción de seguridad, trabajo en equipo y Transiciones entre servicios/unidades.

Cuando se revisa investigaciones locales, los resultados fueron similares. Una investigación realizada en Bogotá ${ }^{26}$, reportó que la percepción de seguridad del paciente fue catalogada como favorable por el $75 \%$ de los trabajadores evaluados, el $23 \%$ piensa que el nivel de seguridad del paciente es adecuado y el $2 \%$ lo cataloga como oportunidad de mejora. Las dimensiones percibidas como fortaleza fueron Aprendizaje organizacional/mejoras continuas (75\%) y comunicación e información sobre errores (77\%); la dimensión catalogada como oportunidad de mejora fue respuesta no punitiva al error (44\%).

En Colombia la normatividad regula y obliga a las instituciones de salud a mejorar sus procesos en relación con la calidad y prevención de eventos adversos ${ }^{27}$. Dentro de las actividades de mejoramiento de la calidad, en Colombia permanentemente se capacita al personal en temas relacionados con calidad y seguridad del paciente, lo que constituye una línea de base mínima para estructurar los programas de seguridad, que permiten a la organización avanzar en procesos como acreditación, en el que la seguridad del paciente es el tema central.

Los diferentes estudios publicados ponen al descubierto el temor de los trabajadores de salud al reporte de los eventos adversos, lo que evidencia que la búsqueda de culpables no ha favorecido la disminución de los errores y que, por el contrario, ha socavado la cultura del registro y la comunicación de los eventos adversos. Un estudio realizado en los Estados Unidos ${ }^{28}$ encontró que las restricciones a la libre expresión del personal de salud, se reducen a la creencia de que los errores que se cometen y se reportan, se mantienen vigentes en sus registros profesionales personales y esto afecta de alguna manera su hoja de vida; así mismo, el personal cree que pueden estar en riesgo de algún castigo si informan de algún evento adverso durante la atención de salud del paciente. Por tal razón, se debe trabajar para crear una cultura justa y adecuada en las diferentes instituciones, que aliente a todos los trabajadores de la salud a informar los incidentes, eventos y errores presentados.

La dimensión comunicación abierta y retroalimentación también es considerada como debilidad en los estudios publicados. Las instituciones deben incluir dentro de sus estrategias el fortalecimiento de la comunicación y el trabajo en equipo, ya que se ha documentado que los colaboradores se ven muy afectados tanto física como psicológicamente al presentarse un evento adverso, debido a la ausencia de estrategias para el afrontamiento de la situación, además de un sentimiento de incompetencia, insuficiencia o culpabilidad y la ausencia de apoyo por parte de su equipo de trabajo o la institución.

El presente estudio tiene algunas limitaciones, como, los resultados del estudio evidencian la percepción del personal de las unidades quirúrgicas la cual puede estar influenciada por aspectos propios del sujeto como motivación, sentido de pertenencia e incluso el estado emocional los cuales, pueden influir al momento de responder el cuestionario; además, el personal que no participó podría tener una percepción diferente de clima de seguridad. También es necesario reconocer que los puntajes bajos de clima de seguridad no representan necesariamente instituciones menos seguras. Se requieren estudios que correlacionen estas mediciones con indicadores como la frecuencia o la severidad de los eventos adversos.

\section{Conclusiones}

El presente estudio reportó una medición de clima de seguridad del paciente con una encuesta validada para Colombia en las unidades quirúrgicas, esta es una oportunidad que ayuda a las instituciones a la obtención de información con mayor confiabilidad para la toma de decisiones. 
Los trabajadores percibían como positivo el proceso de mejoramiento continuo del programa de seguridad, pero sienten que son juzgados al momento de reportar algún evento adverso; esto probablemente esté relacionado con los resultados de la percepción negativa de la dimensión respuesta no punitiva al error. Los eventos adversos deben ser vistos desde una perspectiva sistémica mas no individual. Los colaboradores sienten que las estrategias implementadas por las instituciones son punitivas; otra de las sensaciones que queda en el trabajador es la de no recibir el apoyo suficiente y sentirse señalados por su equipo de trabajo.

Los trabajadores relacionan la sobrecarga laboral con el aumento de incidentes o eventos adversos al calificar esta dimensión negativamente. Por tal razón, es importante que las instituciones busquen estrategias para optimizar los procesos asistenciales y administrativos, y de esta manera fortalecer la política de seguridad. La ausencia de una comunicación eficaz y efectiva pone en riesgo las practicas seguras implementadas por las instituciones. Además, socializar oportunamente los errores y eventos adversos ocurridos a cada equipo de salud involucrado, facilita la percepción de una cultura justa, y el equilibrio entre la actuación profesional y lo no punitivo.

Los factores como el tipo de contratación y el cargo se asocian más a la percepción negativa en las dimensiones comunicación abierta y retroalimentación, transiciones $\mathrm{y}$ transferencias entre servicios, y respuesta no punitiva al error.

La existencia de una reglamentación en salud en Colombia es una fortaleza para el desarrollo de una cultura de la seguridad, lo que permitirá evidenciar precepciones de clima de seguridad más positivas.

\section{Agradecimientos}

Nuestro más sincero agradecimiento a todos los profesionales de salud que han contribuido a la realización de este estudio respondiendo a las encuestas. También a los colaboradores para la adquisición de datos: Hospital Internacional de Colombia; Fundación Cardiovascular de Colombia; Los Comuneros Hospital Universitario de Bucaramanga y ESE Hospital San Juan de Dios de Floridablanca.

\section{Conflictos de intereses}

Los autores declaran no tener ningún conflicto de interés.

\section{Referencias}

1. Manser T. Teamwork and patient safety in dynamic domains of healthcare: a review of the literature. Acta Anaesthesiol Scand. 2009;53(2): 143-151. doi: 10.1111/j.1399-6576.2008.01717.x

2. Bocanegra-Rivera JC, Arias-Botero JH. Characterization and analysis of adverse events in closed liability cases involving anaesthetists who received legal support from the Colombian Society of Anaesthesia and Resuscitation (S.C.A.R.E.), Colombia, 1993-2012. Rev Colomb Anestesiol. 2016; 44(3): 203-210. doi: 10.1016/j. rca.2016.04.008

3. Winch P, Khan S, Naguib A, Yates A, Rice J, Barry $\mathrm{N}$, et al. Transportation of patients following surgery for congenital heart disease: A process review prompted by the opening of a new hospital. 2014; 7(2): 411-415.

4. Manser T. Fragmentation of patient safety research: A critical reflection of current human factors approaches to patient handover. 2013; 2(3): e33. doi: 10.4081/jphr.2013.e33

5. Ministerio de Salud y Protección Social. Evaluación de la frecuencia de eventos adversos y monitoreo de aspectos claves relacionados con la seguridad del paciente.; 2019. 2020. https://www.minsalud.gov. $\mathrm{co} /$ sites/rid/Lists/BibliotecaDigital/RIDE/DE/CA/ Evaluar-frecuencia-eventos-adversos.pdf

6. Haynes AB, Weiser TG, Berry WR, Lipsitz S, Breizat A, Patchen D, et al. A Surgical safety checklist to reduce morbidity and mortality in a global population. N Engl J Med. 2009; 360(5): 491-499. doi: 10.1056/nejmsa0810119

7. Haynes AB, Weiser TG, Berry WR, Lipsitz S, Breizat A, Patchen D, et al. Changes in safety attitude and relationship to decreased postoperative morbidity and mortality following implementation of a checklist-based surgical safety intervention. BMJ Qual Saf. 2011; 20(1): 102-107. doi: 10.1136/ bmjqs.2009.040022

8. Sen AC, Morrow DF, Balachandran R, Du X, Gauvreau K, Jagannath B, et al. Postoperative infection in developing world congenital heart surgery programs: Data from the international quality improvement collaborative. Circ Cardiovasc Qual Outcomes. 2017; 10(4). e002935. doi: 10.1161/ CIRCOUTCOMES.116.002935

9. Bonifield J, Cohen E. 10 errores médicos impactantes. CNN health. 2012; https://edition.cnn. com/2012/06/09/health/medical-mistakes/index. $\mathrm{html}$ 
10. Larrazabal LA, Jenkins KJ, Gauvreau K, Vida $\mathrm{V}$, Benavidez O, GAitán G, et al. Improvement in congenital heart surgery in a developing country: The Guatemalan experience. Circulation. 2007; 116(17): 1882-1887. doi: 10.1161/ CIRCULATIONAHA.107.695403

11. Palacios Macedo A. Birth of a New Program in Mexico City: The Kardias Experience. Pediatr Card Surg Annu. 2008; 11(1): 7-10. doi: 10.1053/j. pcsu.2007.12.002

12. Turley K, Tyndall M, Turley K, Woo D, Mohr T. Radical Outcome Method. Circulation. 1995; 92(9): 245-249. doi: 10.1161/01.CIR.92.9.245

13. Walker IA, Reshamwalla S, Wilson IH. Surgical safety checklists: do they improve outcomes? $\mathrm{Br}$ J Anaesth. 2012; 109(1): 47-54. doi: 10.1093/bja/ aes 175

14. Zhao P, Li Y, Li Z, Jia P, Zhang L, Zhang M. Use of patient safety culture instruments in operating rooms: A systematic literature review. J Evid Based Med. 2017; 10(2): 145-151. doi: 10.1111/ jebm. 12255

15. Godinho Rigobello MC, Fontenele Lima de Carvalho RE, De Bortoli Cassiani SH, Galon T, Capucho HC, de Deus NN. The climate of patient safety: perception of nursing professionals. Acta Paul Enferm. 2012; 25(5): 728-735. doi: 10.1590/ S0103-21002012000500013

16. Sorra J, Nieva V. Hospital Survey on Patient Safety Culture.; 2004. http://www.ahrq.gov

17. Arias Botero JH, Gomez Arias RD, Segura Cardona AM, Acosta Rodriguez F, Quesada Rico JA, Gil Guillen V. Measuring patient safety climate in operating rooms: Validation of the Spanish version of the hospital survey on patient safety. Heal Serv Manag Res. 2020. doi: 10.1177/0951484820943598

18. for Healthcare Research A. Hospital survey on patient safety culture: User's Guide. 2021. http:// www.ahrq.gov

19. Ministerio de la protección social. lineamientos para la implementación de la política de seguridad del paciente. 2008. https://www.minsalud.gov. $\mathrm{co} / \mathrm{sites} / \mathrm{rid} /$ Lists/BibliotecaDigital/RIDE/DE/ CA/LINEAMIENTOS_IMPLEMENTACION_ POLITICA_SEGURIDAD_DEL_PACIENTE.pdf

20. Sanchis DZ, Haddad M do CFL, Girotto E, Silva AMR. Patient safety culture: perception of nursing professionals in high complexity institutions. Rev Bras Enferm. 2020; 73(5): e20190174. doi: 10.1590/0034-7167-2019-0174

21. Abreu IM de, Rocha RC, Avelino FVSD, Guimarães DBO, Nogueira LT, Madeira MZ de A. Cultura de segurança do paciente em centro cirúrgico: visão da enfermagem. Rev Gauch Enferm. 2019; 40(spe): e20180198. doi: 10.1590/19831447.2019.20180198

22. Behzadifar M, Behzadifar M, Jahanpanah F, Bragazzi NL. Patient safety culture assessment in Iran using the "Hospital survey on patient safety culture" tool: A systematic review and metaanalysis. Clin Epidemiol Glob Heal. 2019; 7(4): 641-647. doi: 10.1016/j.cegh.2019.02.008

23. Vega-Ramírez FA, Salazar JG, López-Liria R. Perception of patient's safety culture in primary care nurses. Psychol Soc Educ. 2020; 12(2): 49-60. doi: 10.25115/psye.v10i1.2811

24. Mella Laborde M, Gea Velázquez MT, Aranaz Andrés JM, Ramos Forner G, Compañ Rosique AF. Analysis of the patient safety culture in a university hospital. Gac Sanit. Published online, 2019. doi: 10.1016/j.gaceta.2018.10.004

25. Jaraba Becerril C, Sartolo Romeo MT, Villaverde Royo M V., Albas LE, Jiménez MR. Evaluación de la cultura sobre seguridad del paciente entre médicos residentes de Medicina familiar y comunitaria en un servicio de urgencias hospitalario. An Sist Sanit Navar. 2013; 36(3): 471-477. doi: 10.4321/s113766272013000300012

26. Palencia Guerra CD, González Gutiérrez NF, Salamanca Díaz LM, Medición de la cultura de seguridad del paciente en un hospital de segundo nivel, Cundinamarca 2018. Agency Healthc Res Qual. Published online 2018. https://repository.ucc. edu.co/handle/20.500.12494/7138

27. Ministerio de Salud y Protección Social. Sistema Obligatorio de Garantía de Calidad En Salud (SOGCS). 2016. https://www.minsalud.gov.co/ salud/PServicios/Paginas/sistema-obligatoriogarantia-calidad-SOGC.aspx

28. Paterick ZR, Paterick BB, Waterhouse BE, Paterick TE. The challenges to transparency in reporting medical errors. J Patient Saf. 2009; 5(4): 205-209. doi: 10.1097/PTS.0b013e3181be2a88 